\title{
Contact angle dependence on the fluid-wall dispersive energy
}

\author{
Martin Horsch, ${ }^{\dagger}$ Martina Heitzig, ${ }^{\ddagger} \perp$ Calin Dan, ${ }^{\mathbb{I}}$ Jens Harting, ${ }^{\S}, \mathbb{I}$ Hans Hasse, $\|$ \\ and Jadran Vrabec*,† \\ Thermodynamics and Energy Technology Laboratory (ThEt), University of Paderborn, Warburger \\ Str. 100, 33098 Paderborn, Germany, Institute of Thermodynamics and Thermal Process \\ Engineering (ITT), University of Stuttgart, Pfaffenwaldring 9, 70569 Stuttgart, Germany, Institute \\ for Computational Physics (ICP), University of Stuttgart, Pfaffenwaldring 27, 70569 Stuttgart, \\ Germany, Faculteit Technische Natuurkunde (TN), Eindhoven University of Technology, P. O. Box \\ 513, 5600 MB Eindhoven, The Netherlands, and Laboratory of Engineering Thermodynamics \\ (LTD), University of Kaiserslautern, Erwin-Schrödinger-Str. 44, 67663 Kaiserslautern, Germany \\ E-mail: jadran.vrabec@upb.de
}

\begin{abstract}
Vapor-liquid menisci of the truncated and shifted Lennard-Jones fluid between parallel planar walls are investigated by molecular dynamics simulation. Thereby, the characteristic energy of the unlike dispersive interaction between fluid molecules and wall atoms is systematically varied to determine its influence on the contact angle. The temperature is varied as
\end{abstract}

\footnotetext{
${ }^{*}$ To whom correspondence should be addressed

${ }^{\dagger}$ University of Paderborn

$\ddagger$ University of Stuttgart (ITT)

"University of Stuttgart (ICP)

${ }^{\S}$ Eindhoven University of Technology

"University of Kaiserslautern

${ }^{\perp}$ Current address: Computer Aided Process-Product Engineering Center (CAPEC), Technical University of Denmark, Søltofts Plads, 2800 Kgs. Lyngby, Denmark
} 
well, covering most of the range between the triple point temperature and the critical temperature of the bulk fluid. The transition between obtuse and acute angles is found to occur at a temperature-independent magnitude of the fluid-wall dispersive interaction energy. On the basis of the present simulation results, fluid-wall interaction potentials can be adjusted to contact angle measurements.

\section{Introduction}

A major challenge for molecular modeling consists in the definition of unlike interaction potentials. In the past, a variety of combination rules were proposed, none of which was found to be valid in general. Several of these, including the Lorentz-Berthelot combination rule, are considered to be a good starting point for further adjustment in most cases 1 . The present work contributes to understanding the dispersive interaction between a solid wall and a fluid, which is essential for the analysis of adsorption and microscopic flow properties.

In principle, the Lorentz-Berthelot rule can be applied for effective pair potentials acting between the fluid particles and the atoms of a solid wall ${ }^{23}$, based on size and energy parameters derived from properties of the solid and the fluid. However, while using combination rules to extrapolate from homogeneous bulk solid and fluid properties to interfacial phenomena can lead to a good agreement with the actual behavior ${ }^{2}$, this approach has only shaky theoretical foundations ${ }^{4}$. Unlike pair potentials between a fluid and a solid can only be expected to give reliable results if they are developed using actual information on fluid-wall contact effects. Since adsorption in nanopores can be studied on the basis of effective pair potentials $\frac{3.5}{4}$, it is obversely possible to fit model parameters to adsorption isotherms $\frac{10}{10}$. The present study follows the line of research, suggested by WERDER et al. 11 , of adjusting unlike parameters to contact angle measurements.

Using density functional theory, TELETZKE et al. 12 examined the dependence of wetting and drying transitions on characteristic size and energy parameters of the fluid-wall dispersive interaction. Subsequently, SOKOŁOWSKI and FISCHER ${ }^{13}$ as well as GIOVAMBATTISTA et al. ${ }^{14}$ investigated fluid density profiles in extremely narrow channels for several values of the fluid-wall 
dispersive energy and surface polarity, respectively. On the microscopic and the nanoscopic level, the statics and dynamics of fluids under confinement and the corresponding three-phase contact lines can also be investigated by the lattice Boltzmann method ${ }^{15}-18$.

Molecular dynamics (MD) simulation can be applied to this problem as well, leading to a consistent molecular approach. The increase in computing power and the development of massively parallel MD software provide tools for simulating systems with a large number of particles. System sizes accessible to MD simulation are getting closer to the smallest experimental settings. This allows comparing simulation data directly to experimental observables for a growing spectrum of properties, including the contact angle. The truncated and shifted Lennard-Jones (LJTS) potential $^{19}$ is used in the present work for describing both the fluid-fluid and the fluid-wall interaction, leading to systems that extend previous studies on interface properties for the LJTS fluid $20-22$. Hysteresis ${ }^{23}$ as well as the formation and growth of liquid precursor layers on the surface ${ }^{4}$ are not discussed as the present work deals with equilibrium properties of the phase boundary only.

\section{Simulation method}

Like the original Lennard-Jones $(\mathrm{LJ})$ potential $u^{\mathrm{LJ}}(r)=4 \varepsilon\left[(\sigma / r)^{12}-(\sigma / r)^{6}\right]$, the LJTS model ${ }^{19}$

$$
u_{i j}^{\mathrm{ts}}\left(r_{i j}\right)= \begin{cases}u^{\mathrm{LJ}}\left(r_{i j}\right)-u^{\mathrm{LJ}}\left(r_{\mathrm{c}}\right) & r_{i j}<r_{\mathrm{c}} \\ 0 & r_{i j} \geq r_{\mathrm{c}}\end{cases}
$$

with a cutoff radius of $r_{\mathrm{c}}=2.5 \sigma$, accurately reproduces the thermophysical properties of several non-polar fluids, in particular noble gases and methane, when adequate values for the size and energy parameters $\sigma$ and $\varepsilon$ are specified 20 . Due to the relatively small cutoff radius, molecular simulation is comparably fast, while the full descriptive power of the LJ potential is retained even for systems with phase boundaries 20 .

In order to accurately describe properties of a solid material, it is usually necessary to employ multi-body potentials which have a large number of model parameters and are computationally 
quite expensive $\mathrm{e}^{24-26}$. The present study, however, does not regard the properties of a specific wall material but rather the influence of the fluid-wall dispersive interaction on the fluid itself. Accordingly, a layered wall was represented here by a comparably straightforward system of coupled harmonic oscillators, using different spring constants $D_{y}$ and $D_{x z}$ for the transverse vibration with respect to the layers

$$
u_{i}^{y}\left(y_{i}\right)=\frac{D_{y}}{2}\left(y_{i}-y_{i}^{\circ}\right)^{2}
$$

wherein $y_{i}^{\circ}$ is the equilibrium value of the $y$ coordinate (i.e. the direction perpendicular to the wall), and longitudinal oscillations,

$$
u_{i j}^{x z}\left(r_{i j}\right)=\frac{D_{x z}}{2}\left(r_{i j}-A\right)^{2}
$$

with respect to the equilibrium bond length $A$ between neighboring atoms $i$ and $j$.

Fluid-wall interactions can be represented by full ${ }^{[5}$ or slightly modified ${ }^{27}$ LJ potentials, acting between fluid particles and the atoms of the solid. Following this approach, the LJTS potential with the size and energy parameters $\sigma_{\mathrm{fw}}=\sigma$ as well as

$$
\varepsilon_{\mathrm{fw}}=\zeta \varepsilon
$$

was applied for the unlike interaction using the same cutoff radius as for the fluid. Potential parameters for the molecular models of the fluid as well as the solid component were chosen such as to represent methane and graphite, respectively. For the fluid, the LJTS size and energy parameters $\sigma=3.7241 \AA$ and $\varepsilon / k=175.06 \mathrm{~K}$, as well as the molecular mass $m=16.04 \mathrm{u}$ were used ${ }^{20}$, so that the carbon-carbon $(\mathrm{C}-\mathrm{C})$ bond length in graphite $A=1.421 \AA^{28}$ can be expressed in LJ units as $A$ $=0.3816 \sigma$, while the interlayer distance $Y=3.35 \AA$ corresponds to $0.8996 \sigma$.

The spring constant $D_{x z}=15600 \mathrm{~N} / \mathrm{m}$ related to the $\mathrm{sp}^{2}$ bonds was adjusted to the $\mathrm{C}-\mathrm{C}$ radial distribution function obtained from simulations with the $\operatorname{TERSOFF}^{24}$ potential. This distribution

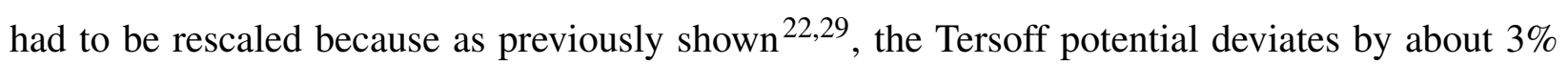
from the actual bond length in graphite. In agreement with the relation between the $\mathrm{C}-\mathrm{C}$ bond energy $(4.3 \mathrm{eV})$ and the interaction energy between adjacent graphite layers $(0.07 \mathrm{eV})^{30}$, the in- 

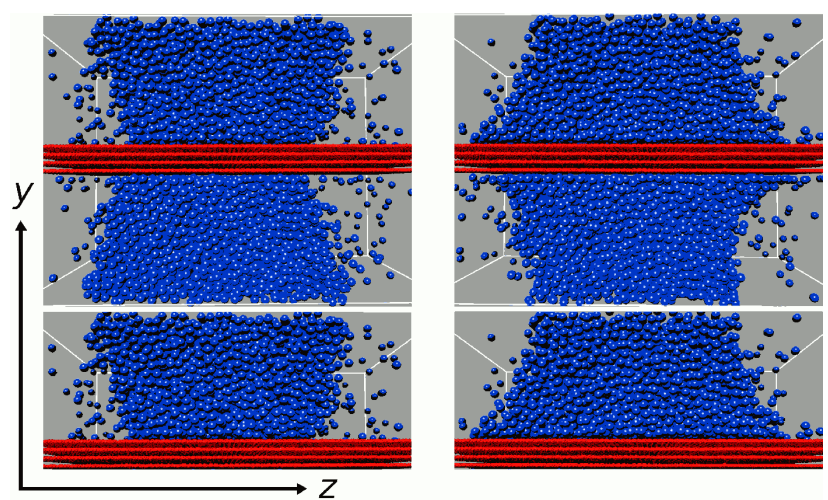

Figure 1: Simulation snapshots for the reduced fluid-wall dispersive energy $\zeta=0.09$ (left) and 0.16 (right) at a temperature $T=0.73 \varepsilon / k$. The upper half is reproduced in the bottom to illustrate the effect of the periodic boundary condition.

terlayer spring constant was specified as $D_{y}=D_{x z} / 60$. Equations $(2)$ and $(3)$ ensure that the wall atoms oscillate around the fixed $y$ coordinate that corresponds to their layer, while no particular $x$ and $z$ coordinates are preferred because the atoms are only connected with their immediate neighbors to permit individual sliding of the wall layers.

Massively parallel canonical ensemble MD simulations were conducted with the program $\ell s 1$ $\operatorname{Mardy} n^{31}$ to obtain the contact angle dependence on the temperature and the reduced fluid-wall dispersive energy. For all simulation runs, the integration time step was specified as $1 \mathrm{fs}$. Vapor and liquid were independently equilibrated in homogeneous simulations for $10 \mathrm{ps}$. This was followed by 200 ps of equilibration for the combined system, i.e. a liquid meniscus surrounded by vapor, with a wall consisting of four to seven layers, cf. Figure 1, where the starting configuration contained a planar vapor-liquid interface perpendicular to the $z$ coordinate axis. Note that the distance from the wall is given by the $y$ coordinate, while $z$ is the characteristic direction for the density gradient of the fluid. The periodic boundary condition was applied to the system, leaving a channel for the fluid with a height of $27 \sigma$ between the wall and its periodic image.

Via binning, the density profiles were averaged over at least 800 ps after equilibration. The arithmetic mean density $\rho_{\mathrm{vl}}=\left(\rho^{\prime}+\rho^{\prime \prime}\right) / 2$ was applied to define the position of the phase boundary, where $\rho^{\prime}$ and $\rho^{\prime \prime}$ are the saturated bulk densities of liquid and vapor which are known for the LJTS fluid from previous work ${ }^{20}$. In the immediate vicinity of the wall, the fluid is affected by short- 


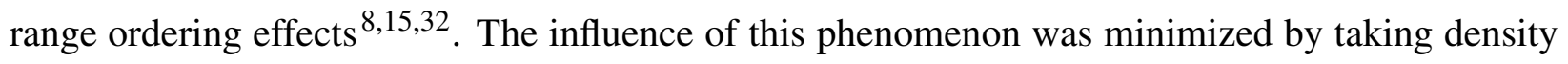
averages over a bin size of about $1 \sigma$, cf. Figure 2 , following GiovAmBATTISTA et al. ${ }^{33}$. A circle was adjusted to the positions of the interface in the bins corresponding to distances between 3 and $11 \sigma$ from the wall, and the tangent to this circle at a distance of $1 \sigma$ from the wall was consistently used to determine the contact angle.

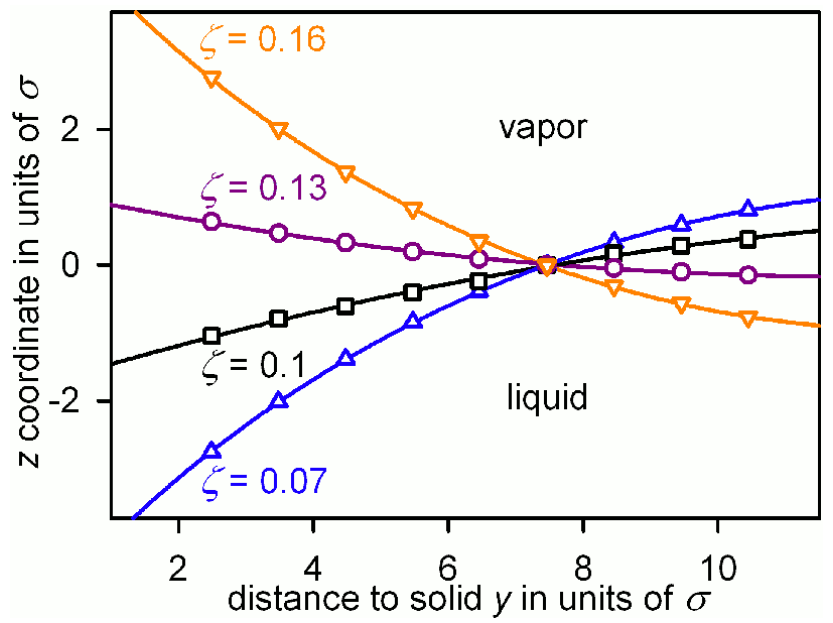

Figure 2: Vapor-liquid interface profiles for the reduced fluid-wall dispersive energy $\zeta=0.07$ (upward triangles), 0.10 (squares), 0.13 (circles), and 0.16 (downward triangles) at a temperature $T$ $=0.82 \varepsilon / k$. Note that the full lines are circle segments, adjusted to the data points that are represented by symbols. The almost perfect match between the individual simulation results, indicated by their collective agreement with the fit, reflects the precision of the present simulation data. At $T=1 \varepsilon / k$, however, the margin of error becomes more significant.

\section{Simulation results}

Menisci between parallel planar walls were simulated for a reduced fluid-wall dispersive energy $\zeta$ between 0.07 and 0.16 at temperatures of $0.73,0.82,0.88$, and $1 \varepsilon / k$. Note that for the bulk LJTS fluid, the triple point temperature is about $0.65 \varepsilon / k$ according to VAN MEEL et al. ${ }^{34}$ while the critical temperature is $1.0779 \varepsilon / k$ according to VRABEC et al. $\frac{20}{}$, so that almost the entire regime of vapor-liquid coexistence was covered here.

High values of $\zeta$ correspond to a strong attraction between fluid particles and wall atoms, leading to a contact angle $\vartheta<90^{\circ}$, i.e. to partial $\left(\vartheta>0^{\circ}\right)$ or perfect $\left(\vartheta=0^{\circ}\right)$ wetting of the 
surface. As expected, with increasing fluid-wall dispersive energy, the extent of wetting grows, cf. Figure 1. As can be seen in Table 1 and Figure 2, the transition from obtuse to acute contact angles occurs at $\zeta$ values between 0.11 and 0.13 over the whole studied temperature range. Present simulation results were correlated by

$$
\cos \vartheta(T, \zeta)=\left(1+\frac{\tau^{1.7}}{27}\right) \tanh \frac{\zeta-Z}{0.087}
$$

where $\tau=\left(1-T / T_{\mathrm{c}}\right)^{-1}$ approaches infinity for $T \rightarrow T_{\mathrm{c}}$, while $Z=0.119$ is the reduced fluid-wall dispersive energy that leads to a contact angle of $\vartheta=90^{\circ}$.

Perfect wetting or drying is present where Eq. (5) yields $\cos \vartheta \geq 1$ or $\cos \vartheta \leq-1$, respectively. In particular, both the value of $Z$ and the symmetry relation

$$
\cos \vartheta(T, Z-\Delta \zeta)=-\cos \vartheta(T, Z+\Delta \zeta)
$$

were found to be temperature-independent. Note that the simulation results stronlgy suggest such a symmetry property, cf. Figure 3 , which is not an artifact of the correlation.

Figure 3 shows, particularly for high temperatures, that there is a narrow range of $\zeta$ values that lead to the formation of a contact angle as opposed to perfect wetting or drying. The present

Table 1: Contact angle of the LJTS fluid on graphite from MD simulation as a function of reduced fluid-wall dispersive energy and temperature.

\begin{tabular}{l|rrrr}
\multicolumn{1}{r|}{$k T / \varepsilon$} & 0.73 & 0.82 & 0.88 & 1 \\
\hline 0.07 & $127^{\circ}$ & $134^{\circ}$ & $139^{\circ}$ & $180^{\circ}$ \\
0.09 & $112^{\circ}$ & $116^{\circ}$ & $119^{\circ}$ & $180^{\circ}$ \\
0.10 & $107^{\circ}$ & $106^{\circ}$ & $109^{\circ}$ & $145^{\circ}$ \\
0.11 & $99^{\circ}$ & $95^{\circ}$ & $96^{\circ}$ & $128^{\circ}$ \\
0.12 & & & $86^{\circ}$ & $86^{\circ}$ \\
0.13 & $82^{\circ}$ & $79^{\circ}$ & $76^{\circ}$ & $81^{\circ}$ \\
0.14 & $73^{\circ}$ & $67^{\circ}$ & $63^{\circ}$ & $0^{\circ}$ \\
0.16 & $58^{\circ}$ & $45^{\circ}$ & $40^{\circ}$ & $0^{\circ}$ \\
\hline
\end{tabular}




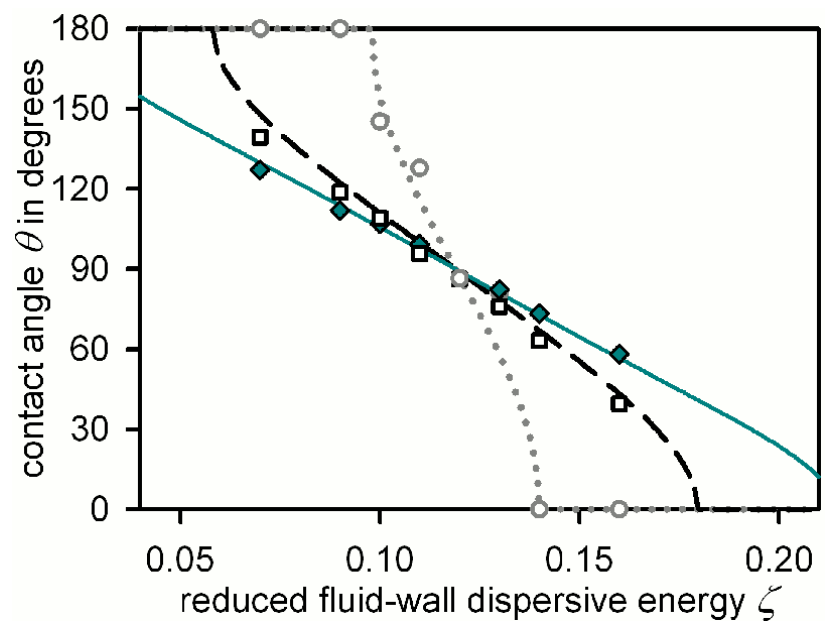

Figure 3: Simulation results and correlation, cf. Eq. (5), for the contact angle in dependence of the reduced fluid-wall dispersive energy $\zeta$ at temperatures $T=0.73$ (diamonds and solid line), 0.88 (squares and dashed line) as well as $1 \varepsilon / k$ (circles and dotted line).

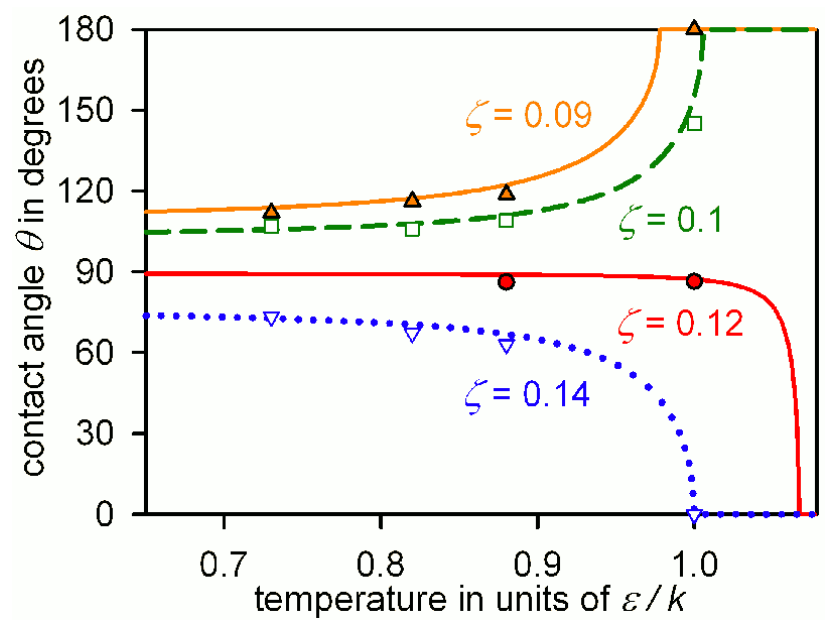

Figure 4: Simulation results and correlation, cf. Eq. (5), for the contact angle in dependence of the temperature at fluid-wall dispersive energies $\zeta=0.09$ (upward triangles and solid line), 0.10 (squares and dashed line), 0.12 (bullets and solid line) as well as 0.14 (downward triangles and dotted line). The entire temperature range between triple point and critical point of the bulk fluid is shown.

plots agree qualitatively with those determined by GiovambatTISTA et al. ${ }^{[33}$ for the influence of the polarity of hydroxylated silica surfaces on the contact angle formed with water. In Figure 4 , it can be seen that the extent of wetting (for $\zeta>Z$ ) or drying (for $\zeta<Z$ ), respectively, increases as the temperature approaches $T_{\mathrm{c}}$. Eventually, this leads to the known phenomenon of critical point wetting ${ }^{35}$ for the whole range above a wetting temperature $T_{\mathrm{w}}$. The effects described above can be 
accounted for by the Young equation

$$
\cos \vartheta(T, \zeta)=\frac{\gamma_{\mathrm{s}}\left(T, \zeta, \rho^{\prime \prime}\right)-\gamma_{\mathrm{s}}\left(T, \zeta, \rho^{\prime}\right)}{\gamma(T)}
$$

which relates the vapor-liquid surface tension $\gamma$ and the contact angle to the interfacial tension $\gamma_{\mathrm{s}}$ that acts between wall and vapor or liquid. The deviation from $\vartheta=90^{\circ}$ increases with $T \rightarrow T_{\mathrm{c}}$ because $\gamma(T)$ converges to zero faster than the density difference $\rho^{\prime}(T)-\rho^{\prime \prime}(T)$ between the two fluid phases. The relevant critical exponents in case of the LJTS fluid are

$$
\lim _{T \rightarrow T_{\mathrm{c}}} \frac{\ln \left[\rho^{\prime}(T)-\rho^{\prime \prime}(T)\right]}{\ln \left(T_{\mathrm{c}}-T\right)} \approx \frac{1}{3}
$$

for the saturated densities of the bulk fluid ${ }^{20}$, in accordance with the GUGGENHEIM ${ }^{36}$ approach, and

$$
\frac{d \ln \gamma(T)}{d \ln \left(T_{\mathrm{c}}-T\right)} \approx 1.21
$$

for the vapor-liquid surface tension ${ }^{20}$, confirming a similar value (1.26) obtained from fluctuation theory of critical phenomena 37 .

The correlation given by Eq. (5) suggests for the present system a first-order transition between partial and perfect wetting or drying, respectively, as described by $\mathrm{CAHN}^{35}$. With $\zeta \approx Z$, Figure 3 shows that the contact angle $\vartheta$ depends linearly on the fluid-wall dispersive energy, and the symmetry property suggests for $\zeta=Z$ that $\gamma_{\mathrm{s}}$ does not depend on the density of the fluid.

\section{Comparative discussion}

Qualitatively, the symmetry relation given by Eq. (6) corroborates MONSON ${ }^{23}$ who obtained the same property based on mean-field DFT calculations. If each layer of the wall is approximated as a plane of uniform density $\eta$, the well depth of the fluid-wall dispersive interaction, which can be 
used to compare different interaction models quantitatively, is given by

$$
W=-\eta \zeta \min _{y>0} \int_{0}^{\infty} d \lambda 2 \pi \lambda \sum_{\ell=0}^{L-1} u^{\mathrm{ts}}\left(\left[(y+\ell Y)^{2}+\lambda^{2}\right]^{1 / 2}\right)
$$

for a system of $L$ layers with an interlayer distance of $Y$. In the present case with $r_{\mathrm{c}}=2.5 \sigma$ for the LJTS potential as well as a surface density of $\eta=5.287 \sigma^{-2}$, an interlayer distance of $Y=0.8996$ $\sigma$, and the number of layers $L=3$ (the fourth layer of the wall is beyond the cutoff radius), one obtains

$$
W=17.29 k T_{\mathrm{c}} \cdot \zeta
$$

normalized by the critical temperature $T_{\mathrm{c}}=1.0779 \varepsilon / k$ of the bulk LJTS fluid 20 . The transition from obtuse to acute contact angles, occurring at $Z=0.119$ in the present case, therefore corresponds to a well depth of $W_{Z}=2.057 k T_{\mathrm{c}}$.

A very similar system was investigated by BUCIOR et al. ${ }^{8}$, albeit for the LJTS fluid with a cutoff radius of $2^{7 / 6} \sigma$ as well as a rigid wall with a surface density $\eta=1.7342 \sigma^{-2}$ and a single solid layer, i.e. $L=1$. For that fluid, the critical temperature is given by SCHRADER et al. $\frac{38}{\text { as }}$ $T_{\mathrm{c}}=0.9999 \varepsilon / k$, so that the fluid-wall dispersive energy was related to the well depth by $W=$ $5.429 k T_{\mathrm{c}} \cdot \zeta$ according to Eq. 10 . From the density profiles of BUCIOR et al. ${ }^{8}$ one finds that a rectangular contact angle is reached for a reduced fluid-wall dispersive energy $\zeta$ between 0.61 and 0.7, i.e. $3.3 k T_{\mathrm{c}}<W_{Z}<3.8 k T_{\mathrm{c}}$ which is on the same order of magnitude as the present result. The quantitative deviation has to be attributed to the different solid structure, since the single wall layer of BUCIOR et al. ${ }^{8}$ leads to a faster decay of the fluid-wall dispersion with respect to the distance than in the present simulations where three layers can directly interact with the fluid. A larger value of $W_{Z} /\left(k T_{\mathrm{c}}\right)$ is required to compensate for the effectively smaller length scale of the dispersive interaction. 
The present correlation, cf. Eq. (5), predicts perfect wetting for

$$
T_{\mathrm{w}}\left(\frac{W}{k T_{\mathrm{c}}}\right)=\left(1-0.144\left[\left(\tanh \left[0.665 \frac{W}{k T_{\mathrm{c}}}-1.37\right]\right)^{-1}-1\right]^{-0.588}\right) T_{\mathrm{c}}
$$

The transition to perfect wetting was also simulated by BoJAN et al. ${ }^{39}$ who applied the Monte Carlo method in the grand canonical ensemble to neon on metal surfaces. Thereby, the full LJ fluid, with $T_{\mathrm{c}}=1.310 \varepsilon / k$ as determined by LOTFI et al. $\stackrel{40}{ }$, was used to model neon. With $W$ $=2.13 k T_{\mathrm{c}}$, representing magnesium, they obtained a wetting temperature of $T_{\mathrm{w}} \approx 0.50 T_{\mathrm{c}}$, as opposed to the present results which imply that perfect wetting is only reached above $0.974 T_{\mathrm{c}}$, in the immediate vicinity of the critical temperature.

As BOJAN et al. $\frac{39}{19}$ themselves remark, their calculations predict a much lower wetting temperature than a similar previous study by SOKOŁOWSKI and FISCHER ${ }^{41}$ on the local structure of fluid argon in contact with solid carbon dioxide (with the same value of $W / \varepsilon$ ). The latter MD simulation results used a size parameter $\sigma_{\mathrm{fw}} / \sigma$ that is significantly smaller than unity in case of argon and carbon dioxide ${ }^{32 / 41}$ and therefore cannot be directly compared to the present work, since varying the interaction length scale can lead to qualitatively different properties such as a change in the order of the wetting transition $\frac{12}{12}$. However, it should be pointed out that significantly better agreement was obtained here with the results of SOKOŁOWSKI and FISCHER ${ }^{41}$ than with those of BOJAN et al. 39 .

The results presented above can now be used to provide an estimate for the magnitude of the dispersive interaction between fluids and wall materials for which experimental data on the contact angle are available. For instance, regarding the refrigerant R134a (1,1,1,2-tetrafluoroethane) at temperatures between 10 and $80^{\circ} \mathrm{C}$, VADGAMA and HARRIS ${ }^{42}$ obtained contact angles of $5.5^{\circ} \pm$ $1^{\circ}$ on copper and $7^{\circ} \pm 1^{\circ}$ on aluminum. With $T=0.85 T_{\mathrm{c}}$, which is $45^{\circ} \mathrm{C}$ for $\mathrm{R} 134 \mathrm{a}$, the well depth can be estimated as $W / k \approx 2.9 T_{\mathrm{c}}=1100 \mathrm{~K}$ in both cases on the basis of Eqs. (5) and (11). 
This can be related to a molecular model of the dispersive interaction by

$$
W=-\rho_{\mathrm{w}} \min _{y>0} \int_{0}^{\infty} d \lambda 2 \pi \lambda \int_{0}^{\infty} d v u_{\mathrm{fw}}\left(\left[(y+v)^{2}+\lambda^{2}\right]^{1 / 2}\right)
$$

wherein $\rho_{\mathrm{w}}$ is the density of the solid wall and $u_{\mathrm{fw}}$ is the dispersive interaction potential acting between a fluid molecule and a wall atom. Note that, while Eq. (10) corresponds to a sum over truncated and shifted LJ-10-4 terms, Eq. (13) does not rely on any particular assumption on the internal structure of the solid wall. If e.g. a LJ-12-6 potential is used for $u_{\mathrm{fw}}$, it corresponds to a LJ-9-3 interaction. This reasoning can plausibly be applied to all fluids that do not exhibit an excessively polar or anisotropic structure.

\section{Conclusion}

The contact angle formed between a wall and a vapor-liquid interface was determined by canonical ensemble MD simulation, where the magnitude of the dispersive fluid-wall interaction and the temperature were varied. Over the whole temperature range under investigation, the contact angle dependence on the fluid-wall dispersive energy was found to follow a simple symmetry law. At a temperature-independent value of the reduced fluid-wall dispersive energy, the interfacial tension between vapor and solid as well as liquid and solid is equal, corresponding to the transition between obtuse and acute contact angles.

\section{Acknowledgement}

The authors thank Deutsche Forschungsgemeinschaft for funding SFB 716, the German Federal Ministry of Education and Research (BMBF) for funding the IMEMO project, and the D-Grid Initiative as well as the High Performance Computing Center Stuttgart (HLRS) for providing access to the $g t 4 b w$ supercomputer (D-Grid resource hlrs-bwgrid). Furthermore, they thank G. K. Auernhammer and M. Sokuler (MPI für Polymerforschung), F. Gähler (Universität Bielefeld), M. Hecht 
(HLRS) as well as G. C. Lehmann (Universität Paderborn) for discussions and M. Buchholz (TU München) as well as M. Bernreuther, D. Jenz, and C. Niethammer (HLRS) for their contribution to developing the $\ell$ s 1 Mardyn program. The presented research was conducted under the auspices of the Boltzmann-Zuse Society of Computational Molecular Engineering (BZS).

\section{References}

(1) Schnabel, T.; Vrabec, J.; Hasse, H. J. Mol. Liq. 2007, 130, 170-178.

(2) Steele, W. A. J. Phys. Chem. 1978, 82, 817-821.

(3) Schapotschnikow, P.; Pool, R.; Vlugt, T. J. H. Comp. Phys. Comm. 2007, 177, 154-157.

(4) Dzyaloshinskii, I. E.; Lifshitz, E. M.; Pitaevskii, L. P. Adv. Phys. 1961, 10, 165-209.

(5) Steele, W. A. The Interaction of Gases with Solid Surfaces; Pergamon: Oxford, 1974.

(6) Findenegg, G. H.; Fischer, J. Faraday Discuss. Chem. Soc. 1975, 59, 38-45.

(7) Fischer, J.; Bohn, M.; Körner, B.; Findenegg, G. H. Chem. Ing. Techn. 1982, 54, 763.

(8) Bucior, K.; Yelash, L.; Binder, K. Phys. Rev. E 2009, 79, 031604.

(9) Schapotschnikow, P.; Hommersom, B.; Vlugt, T. J. H. J. Phys. Chem. C 2009, 113, 1269012698.

(10) Vishnyakov, A.; Ravikovitch, P. I.; Neimark, A. V. Langmuir 1999, 15, 8736-8742.

(11) Werder, T.; Walter, J. H.; Jaffe, R. L.; Halicioglu, T.; Koumoutsakos, P. J. Phys. Chem. B 2003, 107, 1345-1352.

(12) Teletzke, G. F.; Scriven, L. E.; Davis, H. T. J. Chem. Phys. 1982, 78, 1431-1439.

(13) Sokołowski, S.; Fischer, J. J. Chem. Soc. Faraday Trans. 1993, 89, 789-794.

(14) Giovambattista, N.; Rossky, P. J.; Debenedetti, P. G. Phys. Rev. E 2006, 73, 041604. 
(15) Harting, J.; Kunert, C.; Herrmann, H. J. Europhys. Lett. 2006, 75, 328-334.

(16) Diotallevi, F.; Biferale, L.; Chibbaro, S.; Puglisi, A.; Succi, S. Phys. Rev. E 2008, 78, 036305.

(17) Diotallevi, F.; Biferale, L.; Chibbaro, S.; Pontrelli, G.; Succi, S.; Toschi, F. Eur. Phys. J. Spec. Top. 2009, 166, 111.

(18) Spencer, T. J.; Hollis, A. P.; Halliday, I.; Care, C. M. Proceedings of the 2nd Micro and Nano Flows Conference, London, 2009, ISBN 978-1902316734.

(19) Allen, M. P.; Tildesley, D. J. Computer Simulation of Liquids; Clarendon: Oxford, 1987.

(20) Vrabec, J.; Kedia, G. K.; Fuchs, G.; Hasse, H. Mol. Phys. 2006, 104, 1509-1527.

(21) Horsch, M.; Vrabec, J.; Hasse, H. Phys. Rev. E 2008, 78, 011603.

(22) Horsch, M.; Vrabec, J.; Bernreuther, M.; Hasse, H. Proceedings of the 6th International Symposium Turbulence, Heat and Mass Transfer, 2009, ISBN 978-1-56700-262-1.

(23) Monson, P. A. Langmuir 2008, 24, 12295-12302.

(24) Tersoff, J. Phys. Rev. Lett. 1988, 61, 2879-2882.

(25) Engin, C.; Sandoval, L.; Urbassek, H. M. Modelling Simul. Mater. Sci. Eng. 2008, 16, 035005.

(26) Ghiringhelli, L. M.; Valeriani, C.; Los, J. H.; Meijer, E. J.; Fasolino, A.; Frenkel, D. Mol. Phys. 2008, 106, 2011-2038.

(27) Dong, T.; Yang, Z.; Wu, H. Energy Convers. Manag. 2006, 47, 2178-2191.

(28) Wiberg, N. Holleman-Wiberg Inorganic Chemistry; Academic Press: San Diego, 2001.

(29) Kelires, P. C. Phys. Rev. B 1993, 47, 1829-1839.

(30) Cotton, F. A.; Wilkinson, G.; Gaus, P. L. Basic Inorganic Chemistry; Wiley: Chichester, 1994. 
(31) Bernreuther, M.; Niethammer, C.; Horsch, M.; Vrabec, J.; Deublein, S.; Hasse, H.; Buchholz, M. Innovatives Supercomputing in Deutschland 2009, 7, 50-53.

(32) Kozak, E.; Sokołowski, S. J. Chem. Soc. Faraday Trans. 1991, 87, 3415-3422.

(33) Giovambattista, N.; Debenedetti, P. G.; Rossky, P. J. J. Phys. Chem. B 2007, 111, 9581-9587.

(34) van Meel, J. A.; Page, A. J.; Sear, R. P.; Frenkel, D. J. Chem. Phys. 2008, 129, 204505.

(35) Cahn, J. W. J. Chem. Phys. 1977, 66, 3662-3672.

(36) Guggenheim, E. A. J. Chem. Phys. 1945, 13, 253-261.

(37) Anisimov, M. A. Critical phenomena in liquids and liquid crystals; Nauka: Moscow, 1987.

(38) Schrader, M.; Virnau, P.; Binder, K. Phys. Rev. E 2009, 79, 061104.

(39) Bojan, M. J.; Stan, G.; Curtarolo, S.; Steele, W. A.; Cole, M. W. Phys. Rev. E 1999, 59, 864-873.

(40) Lotfi, A.; Vrabec, J.; Fischer, J. Mol. Phys. 1992, 76, 1319-1333.

(41) Sokołowski, S.; Fischer, J. Phys. Rev. A 1990, 41, 6866-6870.

(42) Vadgama, B.; Harris, D. K. Exp. Therm. Fluid Sci. 2007, 31, 979-984. 